\section{(6) OPEN ACCESS}

\title{
Disease progression in systemic sclerosis-overlap syndrome is significantly different from limited and diffuse cutaneous systemic sclerosis
}

\author{
Pia Moinzadeh, ${ }^{1}$ Elisabeth Aberer, ${ }^{2}$ Keihan Ahmadi-Simab, ${ }^{3}$ Norbert Blank, ${ }^{4}$ \\ Joerg H W Distler, ${ }^{5}$ Gerhard Fierlbeck, ${ }^{6}$ Ekkehard Genth, ${ }^{7}$ Claudia Guenther, ${ }^{8}$ \\ Ruediger Hein, ${ }^{9}$ Joerg Henes, ${ }^{10}$ Lena Herich, ${ }^{11}$ Ilka Herrgott, ${ }^{12}$ Ina Koetter, ${ }^{13}$ \\ Alexander Kreuter, ${ }^{14}$ Thomas Krieg, $^{1}$ Kathrin Kuhr, ${ }^{11}$ Hanns-Martin Lorenz, ${ }^{15}$ \\ Florian Meier, ${ }^{16}$ Inga Melchers, ${ }^{17}$ Hartwig Mensing, ${ }^{18}$ Ulf Mueller-Ladner, ${ }^{16}$ \\ Christiane Pfeiffer, ${ }^{19}$ Gabriela Riemekasten, ${ }^{20}$ Miklós Sárdy, ${ }^{21}$ Marc Schmalzing, ${ }^{10}$ \\ Cord Sunderkoetter, ${ }^{12}$ Laura Susok $^{22}$ Ingo H Tarner, ${ }^{16}$ Peter Vaith, ${ }^{23}$ \\ Margitta Worm, ${ }^{24}$ Gottfried Wozel, ${ }^{8}$ Gabriele Zeidler, ${ }^{25}$ Nicolas Hunzelmann, ${ }^{1}$ \\ and all participating DNSS centers
}

\section{Handling editor Tore K Kvien \\ - Additional material is published online only. To view please visit the journal online (http://dx.doi.org/10.1136/ annrheumdis-2013-204487). \\ For numbered affiliations see end of article. \\ Correspondence to Dr Pia Moinzadeh, Department of Dermatology and Venereology, University of Cologne, Kerpener Str. 62, Köln, Cologne 50937 Germany; \\ pia.moinzadeh@uk-koeln.de}

Received 19 August 2013 Revised 12 November 2013 Accepted 10 December 2013 Published Online First 3 January 2014

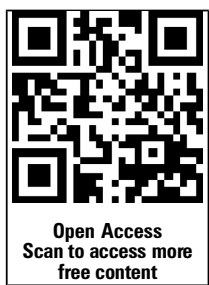

CrossMark

To cite: Moinzadeh $P$, Aberer E, Ahmadi-Simab K, et al. Ann Rheum Dis 2015;74:730-737.

\section{ABSTRACT}

Background Systemic sclerosis (SSC)-overlap syndromes are a very heterogeneous and remarkable subgroup of SSc-patients, who present at least two connective tissue diseases (CTD) at the same time, usually with a specific autoantibody status.

Objectives To determine whether patients, classified as overlap syndromes, show a disease course different from patients with limited SSC (ICSSC) or diffuse cutaneous SSc (dcSSc).

Methods The data of 3240 prospectively included patients, registered in the database of the German Network for Systemic Scleroderma and followed between 2003 and 2013, were analysed.

Results Among 3240 registered patients, 10\% were diagnosed as SSc-overlap syndrome. Of these, $82.5 \%$ were female. SSc-overlap patients had a mean age of 48 \pm 1.2 years and carried significantly more often 'other antibodies' (68.0\%; $p<0.0001)$, including anti-U1RNP, -PmScl, -Ro, -La, as well as anti-Jo-1 and -Ku antibodies.

These patients developed musculoskeletal involvement earlier and more frequently $(62.5 \%)$ than patients diagnosed as ICSSc (32.2\%) or dcSSc $(43.3 \%)$ $(p<0.0001)$. The onset of lung fibrosis and heart involvement in SSc-overlap patients was significantly earlier than in patients with ICSSc and occurred later than in patients with dcSSc. Oesophagus, kidney and $\mathrm{PH}$ progression was similar to lcSSc patients, whereas dcSSc patients had a significantly earlier onset.

Conclusions These data support the concept that SSc-overlap syndromes should be regarded as a separate SSc subset, distinct from ICSSC and dcSSc, due to a different progression of the disease, different proportional distribution of specific autoantibodies, and of different organ involvement.

\section{INTRODUCTION}

Systemic sclerosis (SSc) is a heterogeneous, multisystem, chronic disorder, leading to fibrosis of the skin and many internal organs. To classify patients with established disease, the American College of Rheumatology published in 1980 preliminary criteria. ${ }^{1}$ Currently a subclassification developed by LeRoy et al, this is the most widely used classification system for limited and diffuse SSc in clinical practice, ${ }^{2}$ and is the basis for many registries worldwide. ${ }^{3}$ In these registries, it became apparent that in a sizeable number of patients, symptoms of SSc occur in combination with those of other connective tissue diseases (CTD), ${ }^{4-10}$ also described by some authors as SSc-overlap syndrome. ${ }^{4}{ }^{11-13} \mathrm{Up}$ to now, no firm classification criteria for SSc-overlap syndromes are established, but it is generally considered when musculoskeletal involvement (myositis, arthritis) or clinical signs of other rheumatic diseases are substantially greater than usually found in SSc patients. ${ }^{12}$

Clinical features of overlap syndrome patients are very heterogeneous, and epidemiological studies report divergent frequencies of overlap subgroups as well as of organ manifestations. ${ }^{11} 1415$

Musculoskeletal involvement, including joints, tendons and muscles, is the most frequent clinical feature, highlighting the difference to other SSc forms. Inflammatory joint involvement is reported to be the second most frequent manifestation in patients with musculoskeletal involvement and overlap syndromes. These patients are often identified by typical clinical symptoms (usually limited skin involvement) together with high titres of anticyclic citrullinated peptides (CCP/ACPA) and/or higher rheumatoid factors (RF). ${ }^{16} 17$

All known classification criteria for overlap syndromes include autoantibodies, which are helpful to separate them from other subsets. ${ }^{18-20} \mathrm{PmScl}$ and anti-U1RNP-antibodies are known to be the most common autoantibodies in patients with overlap syndromes. ${ }^{21-24}$

Pakozdi et al reported recently, that $20 \%$ of the patients attending the Centre for Rheumatology at 
the Royal Free Hospital had overlapping features with other rheumatologic diseases, such as polymyositis/dermatomyositis (43\%), systemic lupus erythematosus (SLE) (8\%), Sjögren's syndrome $(17 \%)$ and rheumatoid arthritis $(32 \%){ }^{12}$

It has been always debated whether patients suffering from overlap syndromes should be regarded as a separate entity, or should be included, depending on their skin involvement, in the two main groups of limited (lcSSc) and diffuse SSc (dcSSc) patients.

In this prospective study, it could be shown for the first time that SSc-overlap syndromes should be viewed as a distinct SSc subset.

\section{MATERIALS AND METHODS}

This study involves 3240 patients, registered in the database of the German Network for Systemic Scleroderma (DNSS). The network combines different subspecialties consisting of rheumatologists, dermatologists, pulmonologists and nephrologists from altogether more than 40 clinical centres. The Ethics Committee of the coordinating centre, that is, the Cologne University Hospital, gave a positive vote on the patient information and consent form for the registry. On the basis of this document, all participating centres received the approval of their local ethics committees prior to registering patients.

Patient data, including information about gender, age, autoantibodies, SSc subsets, symptoms and signs, organ involvement, modified Rodnan Skin Score (mRSS) as well as treatments, were recorded on a prospective basis in a database started in $2003^{25-27}$ with a mean follow-up time of $9.5 \pm 0.2$ years (from the time of SSc onset till the last follow-up visit). A significant number of these patients were classified according to the criteria of LeRoy et $a l^{2}$ as having lcSSc or dcSSc. Additionally, a smaller but still considerable number of patients, did not fulfil these criteria, but were registered to follow-up their course of disease. Data of the first Raynaud phenomenon (RP) onset, as well as data of non-RP onset of skin and organ involvement were recorded. Due to the lack of satisfying classification criteria for patients with different forms of overlap syndromes, all patients with more than one CTD were classified as SSc-overlap syndrome in general, including symptoms and signs, autoantibodies and organ manifestations in detail. This information was used to characterise patients with SSc-overlap syndromes.

\section{SSc subsets}

Patients with overlap syndromes were defined as a disease, occurring with clinical features of SSc, according to the ACR criteria, or main SSc-associated symptoms, simultaneously with those typical for other rheumatic diseases. ${ }^{4}$ These patients were often positive for anti-PmScl, -U1RNP, -Jo-1, -Ku, -Ro or -La autoantibodies. Patients with a mixed connective tissue disease (MCTD) were also included in SSc-overlap syndromes, as MCTD combines features of SLE, SSc and myositis, together with the presence of anti-U1RNP-antibodies.

Patients suffering from dcSSc were characterised by a progressive course of disease with an early onset of RP, usually within 1 year of onset of skin changes. They were defined by rapid skin involvement of the trunk, face, proximal and distal extremities and being frequently associated with antitopoisomerase (ATA) antibodies. $^{2}$

LcSSc was defined by skin thickening of the extremities distal to the knee and elbow joints, facial skin and occurrence of RP, which usually appeared many years prior to skin involvement. These patients are often positive for anticentromer-antibodies (ACA). ${ }^{2}$
Patients with undifferentiated SSc were defined as positive RP together with at least one further feature of SSc (typical nailfold capillary alterations, puffy fingers, pulmonary hypertension $(\mathrm{PH})$ ) and/or detectable SSc-specific autoantibodies without fulfilling the ACR criteria for SSc. ${ }^{28}$

Patients with sclerosis sine scleroderma were defined by positive RP, no skin alterations, $\mathrm{PAH}$, cardiac, pulmonary and gastrointestinal involvement. ${ }^{29}$

Within this study, we focused on patients suffering from lcSSc, dcSSc and SSc-overlap syndromes.

\section{Antinuclear autoantibodies (ANAs)}

The antibody measurement was performed in respective laboratories of the participating centres. Serum was routinely analysed in all registered SSc patients at the first visit and repeated as needed. Autoantibodies were subdivided into SSc-specific (ACA, ATA, anti-PmScl, -U1RNP, -Jo1, -Ku antibodies) and SSc nonspecific autoantibodies (anti-Ro and -La antibodies). Missing data were less than $10 \%$.

\section{Organ involvement}

$R P$ was defined by recurrent vasospasms of small digital arterioles/arteries at fingers and/or toes, usually triggered by cold environment. We defined the age of RP onset as the age, at which the RP first appeared. ${ }^{25}$

The first non-RP onset of organ involvement has been considered as the timepoint of first skin or organ manifestation. The onset of skin involvement has been set as onset of SSc.

Skin involvement was evaluated using the modified Rodnan Skin Score (mRSS), which assesses the skin hardening/thickness by manual palpation of 17 body areas on a scale of 0 to 3 .

Pulmonary manifestation includes pulmonary interstitial fibrosis and/or isolated $\mathrm{PH}$. Isolated pulmonary hypertension was defined as clinical evidence of right-heart failure and/or increased mean pulmonary arterial pressure (PAPm $>25 \mathrm{~mm} \mathrm{Hg}$ at rest or PAP $>30 \mathrm{~mm} \mathrm{Hg}$ during exercise), determined by right-heart catheterisation. Echocardiography was used to identify likely PAH (estimated RVSP $>40 \mathrm{~mm} \mathrm{Hg}$ ).

Pulmonary interstitial fibrosis was established when bilateral basal fibrosis occurred, confirmed by chest X-ray and/or highresolution CT scan together with restrictive pulmonary abnormalities on pulmonary function tests (TLC $<80 \%$ ), were found. We defined a normal diffusing capacity of lung for carbon monoxide (DLCO) level, when it was $>75 \%$, and a low level, when it was less than $75 \%$.

Gastrointestinal involvement was defined as gastrointestinal motility disturbance, dysphagia, nausea, malabsorption, oesophageal stenosis, gastro-oesophageal reflux or intestinal pseudo-obstruction.

Kidney involvement was defined as the presence of renal insufficiency encompassing renal insufficiency due to acute renal crisis (creatinine clearance age-related less than $80 \mathrm{~mL} / \mathrm{min}$ ). The diagnosis of proteinuria was fulfilled in cases of albuminuria $>=30 \mathrm{mg} / 24 \mathrm{~h}$ or $>=20 \mathrm{mg} / \mathrm{L}$; proteinuria $>=300 \mathrm{mg} / 24 \mathrm{~h}$ or $>=200 \mathrm{mg} / \mathrm{L}$.

Cardiac disease was defined by heart palpitation, conduction disturbance and/or diastolic dysfunction.

Skeletal muscle disease was defined as proximal muscle weakness and/or atrophy associated with elevated serum muscle enzyme (creatine phosphokinase, CK) levels and/or articular involvement. ${ }^{25}$ The articular involvement included synovitis with swelling, with or without tenderness to palpation in one or more than one joint. The questionnaire also asked for any kind of joint contractures or tendon friction rubs. 
Sicca syndrome was characterised by reduced glandular function, usually causing a dry mouth and dry eyes, while involvement of the masticatory organ was characterised by microstomia, defined as obvious decreased mouth opening clearly detected by the investigators due to the disease and/or fibrosis of the lingual frenulum.

The recommendations for follow up visits and investigations (echocardiography, electrocardiogram, lung function test, etc) are at least once per year.

\section{Statistics}

Differences between the SSc subsets were investigated, using $\chi^{2}$ test for categorical variables and $t$ tests for continuous parameters. To compare the disease progression in the three main subsets Kaplan-Meier analysis with log rank tests was performed. The starting point of the Kaplan-Meier curves was set as SSc onset, which we defined as the time of first non-RP manifestation (onset of skin involvement). The onset of different organ involvements is illustrated within the course of the disease according to our registered follow-up visits.

Additionally, univariate and multiple logistic regression analysis were used to assess the impact of SSc subsets, autoantibody status, age and gender on organ involvement. OR and the corresponding 95\% CI are reported. To investigate the development of DLCO over regular follow-up time, mixed model analysis with backward selection was applied. Time since SSc onset, group membership and their interaction were included as fix factors, and patient ID as a random effect. In order to guard against type I error inflation due to multiple testing, only $\mathrm{p}$ values below 0.001 were considered statistically significant. Statistical analysis was done with PASW Statistics V.18.0 (Chicago: SPSS). Missing data were less than 15\% for organ manifestations and clinical signs. Missing data for autoantibodies are included within table 2 (less than 10\%).

\section{RESULTS}

\section{Patient characteristics of all SSc subsets}

Between the years 2003 and 2012, a total of 3240 patients with SSc had been registered in the DNSS database. Among all registered patients, $49.3 \%$ were diagnosed as lcSSc and 30.8\% with $\mathrm{dcSS}$, $10.0 \%$ with an overlap syndrome, $7.7 \%$ with undifferentiated scleroderma, $0.7 \%$ with sclerosis sine scleroderma, and $1.4 \%$ patients were categorised as others. Within all registered patients, $81.5 \%$ were female; $87.5 \%$ were positive for ANA, $33.7 \%$ had anticentromere antibodies, and $26.4 \%$ were antitopoisomerase antibody (ATA) positive, while the remaining patients had other antibody specificities (32.5\%).

Of all registered patients (first visit), $13.3 \%$ had $\mathrm{PH}, 36.5 \%$ lung fibrosis, 55.1\% suffered from gastrointestinal involvement, 9.9\% had kidney involvement, $12.5 \%$ suffered from heart involvement, $39.1 \%$ from musculoskeletal involvement and $37.5 \%$ from sicca symptoms.

The mean time between the onset of SSc and the first visit/ registration within our database was for all SSc patients $(\mathrm{n}=2522) 7.6 \pm 0.2$ years (SSc diagnosis to first visit), for lcSSc patients $(\mathrm{n}=1236) 8.3 \pm 0.2$ years (SSc diagnosis to first visit),

Table 1 Frequencies and $p$ values $\left(\chi^{2}\right.$ test) of organ manifestations and current symptoms in patients with overlap syndromes, compared to patients with limited systemic sclerosis/diffuse cutaneous SSc (IcSSc/dcSSc)

\begin{tabular}{|c|c|c|c|c|c|}
\hline & \multicolumn{3}{|l|}{ SSc subsets } & \multicolumn{2}{|l|}{$p$ Values } \\
\hline & Overlap s. $(n=325)$ & ICSSc $(n=1598)$ & dcSSc $(n=997)$ & Overlap versus IcSSc & Overlap versus dcSSc \\
\hline Female & 82.5 & 85.9 & 73.1 & 0.122 & 0.001 \\
\hline Male & 17.5 & 14.1 & 26.8 & & \\
\hline Positive family history & 23.1 & 15.9 & 15.0 & 0.039 & 0.029 \\
\hline Age $($ mean $\pm S D)$ & $48 \pm 1.2$ & $52.8 \pm 0.5$ & $48.2 \pm 0.6$ & 0.0001 & 0.847 \\
\hline Erythrocyte sedimentation rate $($ mean \pm SD) & $23 \pm 1.2$ & $17.9 \pm 0.4$ & $23.4 \pm 0.7$ & 0.0001 & 0.734 \\
\hline $\mathrm{DLCO}(\operatorname{mean} \pm \mathrm{SD})$ & $70.5 \pm 1.5$ & $73.5 \pm 0.7$ & $65.3 \pm 0.9$ & 0.064 & 0.005 \\
\hline Modified Rodnan Skin Score (mean \pm SD) & $6.8 \pm 0.4$ & $7.4 \pm 0.2$ & $15.8 \pm 0.3$ & 0.191 & 0.0001 \\
\hline \multicolumn{6}{|l|}{ Organ involvement } \\
\hline Pulmonary hypertension & 10.8 & 12.3 & 18.2 & 0.402 & 0.003 \\
\hline Lung fibrosis & 35.7 & 24.9 & 61.2 & 0.0001 & 0.0001 \\
\hline Oesophagus & 52.0 & 56.2 & 60.8 & 0.065 & 0.013 \\
\hline Kidney & 6.8 & 8.3 & 14.3 & 0.318 & 0.0001 \\
\hline Heart & 13.8 & 9.6 & 18.3 & 0.046 & 0.104 \\
\hline Musculoskeletal system & 62.5 & 32.2 & 43.3 & 0.0001 & 0.0001 \\
\hline Sicca symptoms & 40.0 & 39.5 & 33.8 & 0.703 & 0.049 \\
\hline \multicolumn{6}{|l|}{ Current clinical signs at first visit } \\
\hline Digital ulcers & 18.2 & 23.3 & 33.3 & 0.034 & 0.0001 \\
\hline Synovitis & 22.8 & 11.8 & 14.7 & 0.0001 & 0.004 \\
\hline Dermatogenous contractures & 20.6 & 19.5 & 35.2 & 0.818 & 0.0001 \\
\hline Tendon friction rubs & 8.0 & 5.4 & 10.4 & 0.090 & 0.198 \\
\hline Elevated creatine phosphokinase levels & 17.8 & 6.3 & 10.5 & 0.0001 & 0.001 \\
\hline Muscle weakness & 36.9 & 20.5 & 29.7 & 0.0001 & 0.032 \\
\hline Muscle atrophy & 19.7 & 9.3 & 17.1 & 0.0001 & 0.453 \\
\hline Dysphagia & 51.4 & 56.9 & 60.3 & 0.023 & 0.001 \\
\hline Renal failure & 8.3 & 10.1 & 13.9 & 0.308 & 0.005 \\
\hline Proteinuria & 8.0 & 6.4 & 11.0 & 0.330 & 0.114 \\
\hline
\end{tabular}


Table 2 Detailed autoantibody status of patients with overlap syndromes, compared to patients with IcSSc/dcSSc

\begin{tabular}{|c|c|c|c|c|c|c|c|c|}
\hline \multirow[b]{3}{*}{ Autoantibodies } & \multicolumn{6}{|c|}{ SSc subsets } & \multicolumn{2}{|l|}{ p Values } \\
\hline & \multicolumn{2}{|c|}{$\begin{array}{l}\text { Overlap } \\
\text { s. }(n=325)\end{array}$} & \multicolumn{2}{|c|}{ IcSSc $(n=1598)$} & \multicolumn{2}{|c|}{ dcSSc $(n=997)$} & \multirow[t]{2}{*}{ Overlap versus IcSSc } & \multirow[t]{2}{*}{ Overlap versus dcSSc } \\
\hline & $\%$ & $\mathrm{md}$ & $\%$ & md & $\%$ & $\mathrm{md}$ & & \\
\hline ANAs positive & 92.0 & 3.1 & 87.9 & 4.1 & 89.1 & 4.1 & 0.050 & 0.240 \\
\hline ACA positive & 15.4 & 5.2 & 53.8 & 7.6 & 8.4 & 8.9 & 0.0001 & 0.001 \\
\hline ATA positive & 13.2 & 4.9 & 16.0 & 7.9 & 52.4 & 8.0 & 0.155 & 0.0001 \\
\hline Other Abs & 68.0 & 4.3 & 26.7 & 6.9 & 31.1 & 7.2 & 0.0001 & 0.0001 \\
\hline Other Abs than ACA \& ATA, including & \multicolumn{2}{|c|}{$(n=221)$} & \multicolumn{2}{|c|}{$(n=426)$} & \multicolumn{2}{|c|}{$(n=310)$} & & \\
\hline PmScl & \multicolumn{2}{|l|}{16.7} & \multicolumn{2}{|l|}{5.9} & \multicolumn{2}{|l|}{2.6} & 0.0001 & 0.0001 \\
\hline anti-U1RNP & \multicolumn{2}{|l|}{33.0} & \multicolumn{2}{|l|}{5.4} & \multicolumn{2}{|l|}{3.9} & 0.0001 & 0.0001 \\
\hline Jo-1 & \multicolumn{2}{|l|}{4.1} & \multicolumn{2}{|l|}{0.5} & \multicolumn{2}{|l|}{1.9} & 0.001 & 0.183 \\
\hline $\mathrm{Ku}$ & \multicolumn{2}{|l|}{3.6} & \multicolumn{2}{|l|}{0.5} & \multicolumn{2}{|l|}{2.3} & 0.006 & 0.601 \\
\hline Ro & \multicolumn{2}{|l|}{24.4} & \multicolumn{2}{|l|}{23.5} & \multicolumn{2}{|l|}{27.7} & 0.770 & 0.484 \\
\hline La & \multicolumn{2}{|l|}{10.9} & \multicolumn{2}{|l|}{10.6} & \multicolumn{2}{|l|}{14.2} & 0.894 & 0.294 \\
\hline
\end{tabular}

for dcSSc patients $(\mathrm{n}=857) 7.3 \pm 0.3$ years $(\mathrm{SSc}$ diagnosis to first visit), and for overlap patients $(\mathrm{n}=238) 7.1 \pm 0.5$ years $(\mathrm{SSc}$ diagnosis to first visit).

The mean follow-up time between the SSc diagnosis and the last follow-up visit registered in the DNSS was $9.5 \pm 0.2$ years for all SSc patients $(\mathrm{n}=2539), 10.1 \pm 0.2$ years for $\mathrm{lcSSc}$ patients $(n=1244), 9.2 \pm 0.3$ years for dcSSc patients $(n=862)$ and 9.6 \pm 0.6 years for SSc-overlap syndromes $(n=241)$.

\section{Overlap syndromes}

Within the group of patients with overlap syndromes $(n=325)$, $82.5 \%(268 / 325)$ were women and had a mean age at time of SSc onset of $48 \pm 1.2$ years $(n=323)$ (table 1$) ; 15.4 \%$ of these patients were ACA positive, $13.2 \%$ ATA positive and $68.0 \%$ carried other antibodies (compared to lcSSc (26.7\%) and dcSSc patients $(31.1 \%) ;(p<0.0001))$. These other antibodies consisted of anti-U1RNP (33.0\%), -Ro (24.4\%), -PmScl (16.7\%), -La (10.9\%), - Ku (3.6\%), -Jo1 antibodies (4.1\%) and others (6.3\%) (table 2).

During follow-up, of all 325 patients, more than $92.0 \%$ maintained their initial diagnosis. Patients with SSc-overlap syndromes tended to have more often a positive family history for rheumatological disorders compared to lcSSc and dcSSc patients, and were significantly more frequently treated with corticosteroids $(60.6 \%$ vs $27.3 \%$ (lcSSc) and $44.3 \%$ (dcSSc); $\mathrm{p}<0.0001)$ and immunosuppressive agents $(58.8 \%$ vs $21.0 \%$ $(\mathrm{lcSSc})$ and $41.6 \%(\mathrm{dcSSc}) ; \mathrm{p}<0.0001)$ than other SSc subsets, as published in $2009 .{ }^{26}$ Additionally, this specific subset also had a significantly lower mRSS compared to dcSSc patients (6.8 \pm 0.4 vs $15.8 \pm 0.3 ; \mathrm{p}<0.0001)$, but a very similar mean mRSS to $\mathrm{lcSS}$ c patients $(7.4 \pm 0.2)$ (table 1$)$. Following the criteria of LeRoy for limited and diffuse extension of skin thickening, we had $76.0 \%$ of patients with a limited extension and $14.8 \%$ with a diffuse form of skin thickening. Significantly more female SSc-overlap patients had a limited skin involvement $(87.8 \%$ vs $63.3 \%$; $<<0.0001)$, while significant more male patients suffered from a diffuse skin involvement $(36.7 \%$ vs $12.2 \%$; $\mathrm{p}<0.0001)$. No significant abnormalities for organ manifestations/clinical signs or antibody distribution have been found. Interestingly, significantly less patients with overlap syndromes suffered from digital ulcers $(18.2 \%$ vs $33.3 \%$; $p<0.0001)$ (see online supplementary table S1).

\section{Musculoskeletal involvement}

Patients with overlap syndrome developed significantly earlier and more often musculoskeletal involvement, followed by patients with diffuse and limited SSc (data shown in table 1 and figure 1A). Musculoskeletal manifestation included muscle weakness $(36.9 \%)$, synovitis $(22.8 \%)$, contractures $(2.6 \%)$, muscle atrophy (19.7\%) and elevated CK levels (17.8\%).

Logistic regression analysis revealed, that overlap patients had threefold the risk of developing musculoskeletal involvement, compared to patients with lcSSc (OR 3.2; p <0.001; 95\%-CI 2.5 to 4.2 ), and double the risk compared to dcSSc patients (OR 2.2; $\mathrm{p}<0.001 ; 95 \%-C I 1.6$ to 2.9 ).

We also found that the course of initially elevated CK serum levels decreased substantially over time, especially in overlap and $\mathrm{dcSSc}$ patients (figure $3 \mathrm{~A}$ ).

In a subgroup of patients classified as overlap syndrome with myositis and CK elevation (44/325), we determined the frequency of gender distribution (70.5\% women, $29.5 \% \mathrm{men})$, as well as clinical features; $59.1 \%$ had oesophageal involvement, $38.6 \%$ of patients had lung fibrosis, and $34.1 \%$ suffered from cardiac involvement, while less than $15.0 \%$ had kidney failure or signs for $\mathrm{PH}$. Most of the patients selected for myositis and CK elevation were PmScl positive (22.7\%) followed by $9.1 \%$ anti-U1RNP antibodies, 6.8\% ATA antibodies and 4.5\% ACA, $\mathrm{Ku}$ - and Jo1-antibodies.

\section{Cardiopulmonary involvement}

Lung fibrosis and heart involvement was diagnosed earliest in patients with dcSSc, followed by SSc-overlap and lcSSc patients ( $\log$ rank test $\mathrm{p}<0.0001)$; patients with SSc-overlap syndromes resulted in a clear intermediate position between patients suffering from lcSSc and dcSSc (figures $1 \mathrm{~B}$ and $2 \mathrm{~A}$ ).

Logistic regression analysis revealed that patients with overlap syndromes had a higher risk of developing lung fibrosis compared to lcSSc patients (OR 1.6; p <0.001; 95\%-CI 1.2 to 2.1), and a reduced risk compared to dcSSc patients (OR 0.4; $\mathrm{p}<0.001 ; 95 \%$-CI 0.3 to 0.5$)$. They also had a lower risk of developing lung fibrosis when they had a high DLCO-level (OR $0.9 ; \mathrm{p}<0.001 ; 95 \%$-CI 0.9 to 1.0$)$. The analysis of DLCO within regular follow-up visits showed no significant interaction, for example, the decrease in DLCO over time does not differ between group memberships. However, there was an overall 
A

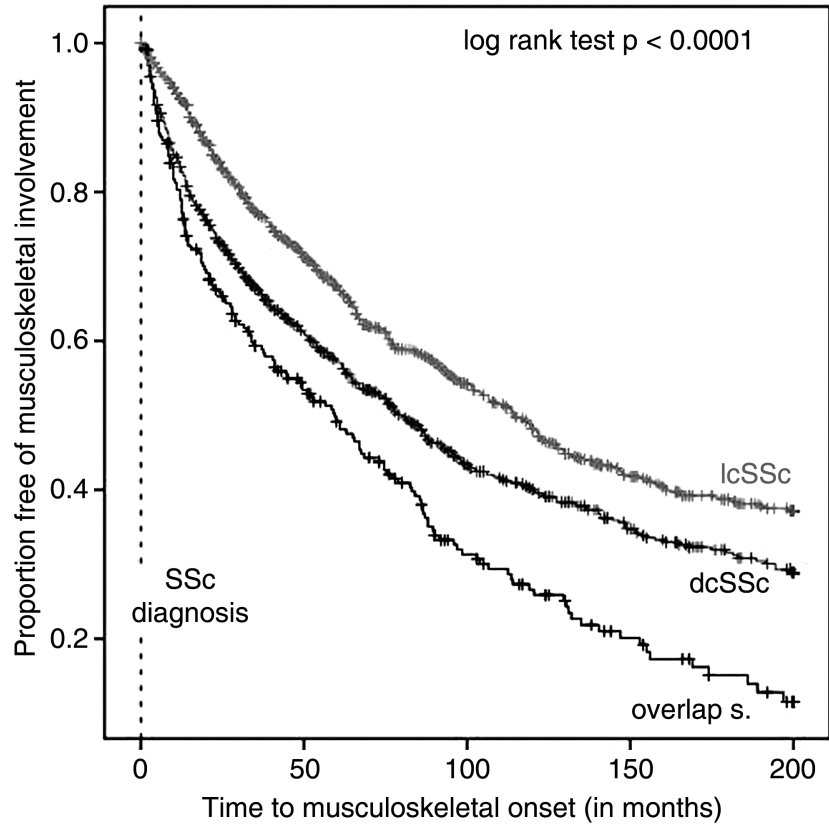

B

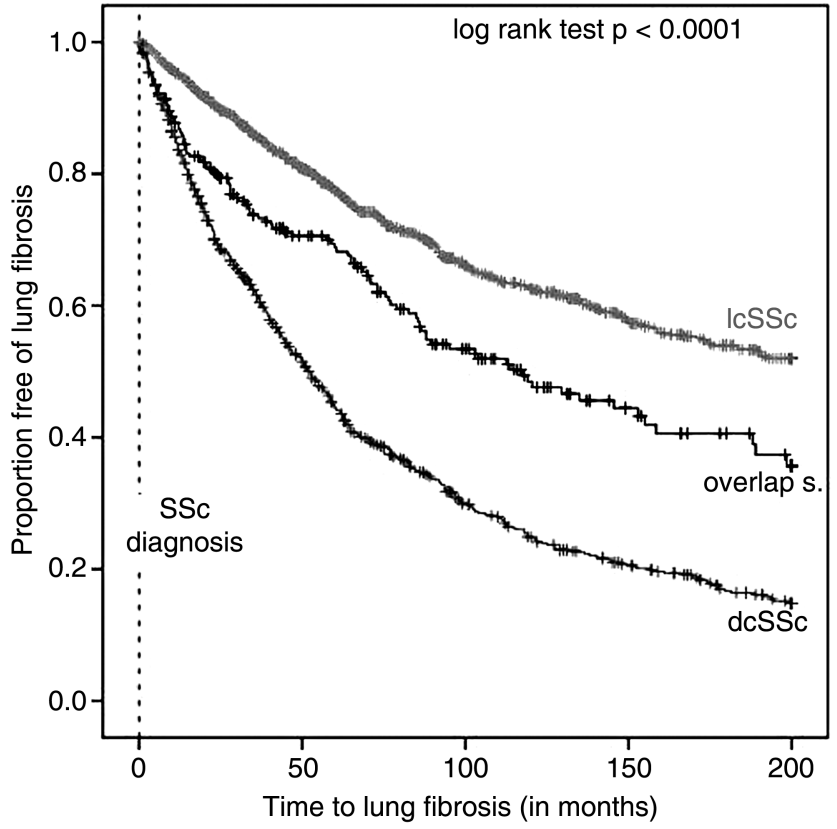

Figure 1 Kaplan-Meier analysis of the onset of (A) musculoskeletal involvement and (B) lung fibrosis in IcSSC, dCSSC and SSc-overlap syndromes. Events (defined as onset of musculoskeletal involvement or lung fibrosis) correspond to step-downs, while censored observations (defined as last follow up visit) are identified as ticks. The plot shows a significant difference $(\mathrm{p}<0.0001)$ between the survival curves of IcSSc, dcSSc and overlap syndrome patients.

significant difference in DLCO between groups $(\mathrm{p}<0.001)$ and between the years since SSc onset $(\mathrm{p}<0.001)$. A posthoc test revealed additionally a significant difference between years 1 and 5 , again the course of the curve of overlap patients was running between dcSSc and lcSSc patients (figure 3B).

\section{Other organ manifestations}

Disease progression, as determined by the onset of $\mathrm{PH}$, oesophagus and kidney involvement of SSc-overlap patients, was similar to lcSSc patients. There was also no significant difference in patients developing gastrointestinal involvement,
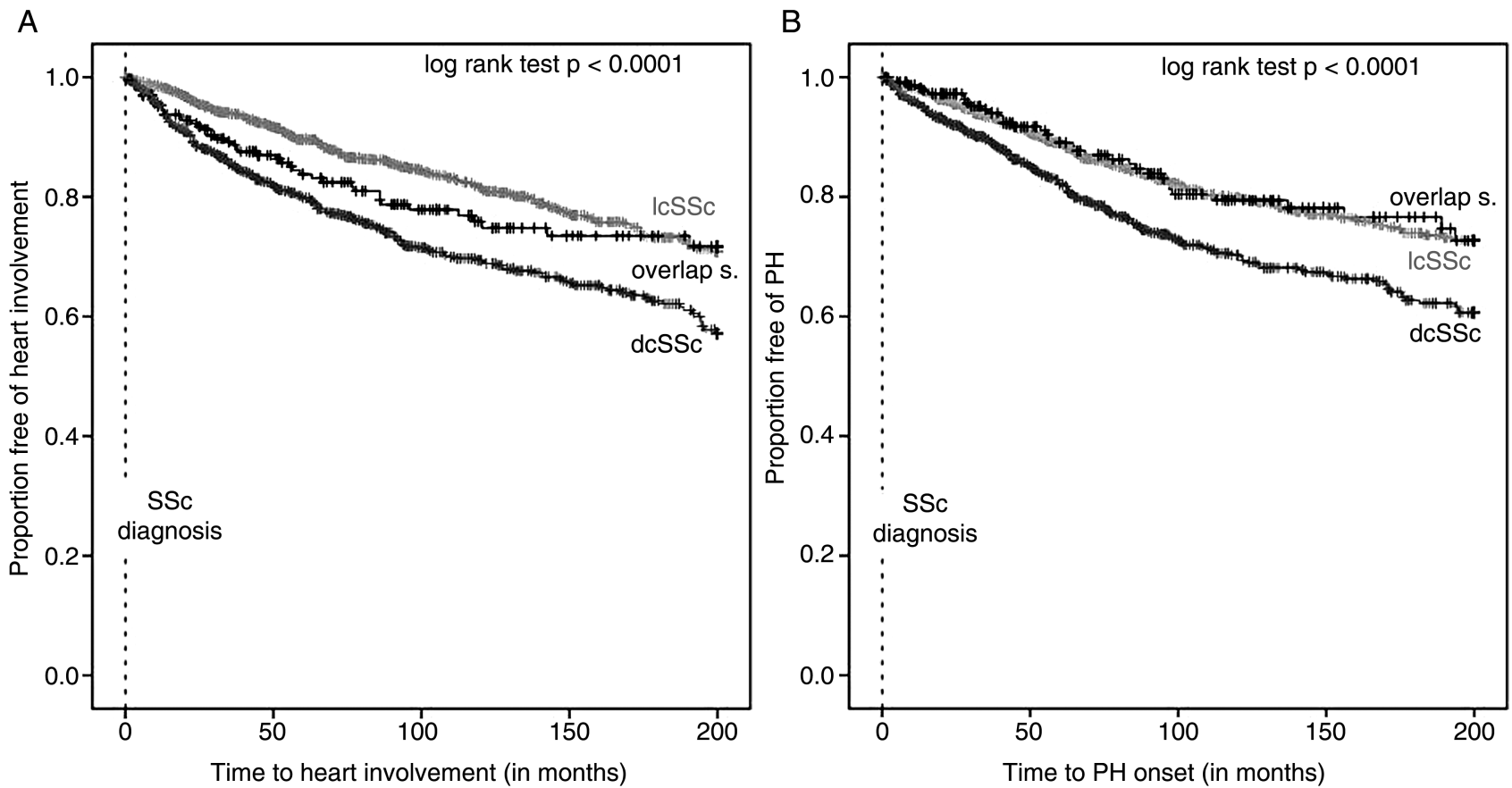

Figure 2 Kaplan-Meier analysis of the onset of (A) heart involvement and (B) PH in IcSSc, dcSSc and SSc-overlap syndromes. Events (defined as onset of heart involvement or PH) correspond to step-downs while censored observations (defined as last follow-up visit) are identified as ticks. The plot shows a significant difference $(p<0.0001)$ between the survival curves of IcSSc, dcSSC and overlap syndrome patients. 
A

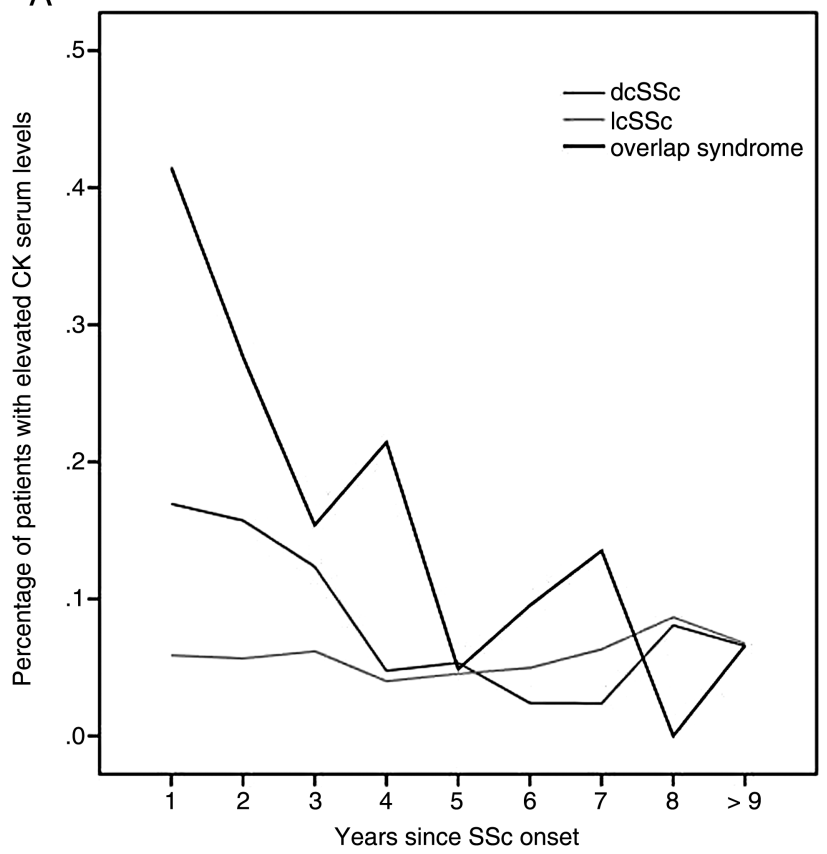

B

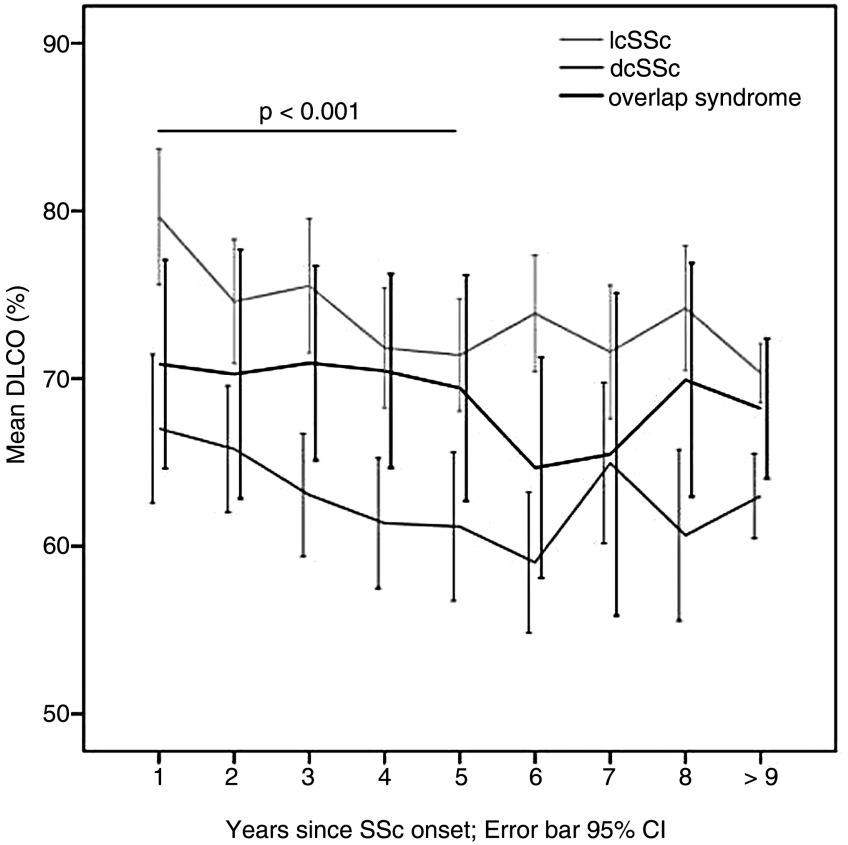

Figure 3 Course of (A) the percentage of patients with elevated CK serum levels and (B) mean DLCO levels across all follow-up visits per year for the three main SSc subsets.

depending on their subset, gender and autoantibody status (table 1 and online supplementary table S1, figure 2B).

\section{Autoantibody status}

Detailed characterisation of overlap patients depending on their autoantibody status revealed that patients with anti-U1RNP-antibodies were significantly younger at RP onset, compared with PmScl-positive patients $(36.2 \pm 1.8$ years vs 44.6 \pm 2.3 years; $p<0.008)$ and tended to be younger at onset of skin manifestations $(39.3 \pm 2.0$ years vs $5.3 \pm 2.4$ years). The interval between RP onset and skin onset was shortest for patients with $\mathrm{Ku}$ antibodies $(0.7 \pm 0.7$ years), followed by Jo1 (1.6 \pm 0.9 years), PmScl (2.0 \pm 0.6 years), ATA (2.1 \pm 0.8 years), Ro $(5.0$ \pm 1.3 years), La (5.2 \pm 2.5 years), anti-U1RNP (6.7 \pm 1.4 years), followed by ACA-positive patients with $11.2 \pm 2.4$ years.

\section{DISCUSSION}

SSc is a heterogeneous disease and includes subsets which are characterised by the extension of skin involvement and circulating autoantibodies. For many years it has been observed, that not all SSc patients fit into the categories defined by LeRoy et $a l^{2} \mathrm{~A}$ considerable number of patients present with symptoms and signs of SSc together with clinical features of other CTDs. ${ }^{5}$

Of the 3240 patients registered in the DNSS, 10.0\% were categorised as overlap syndrome. This frequency is in agreement with other reported data, ranging between $10.0 \%$ and $38.0 \% .^{4} 14^{30-32}$ Our study presents one of the largest studies, characterising patients with overlap syndromes in direct comparison with the two main SSc subsets, and our data indicate that overlap patients clearly differ from lcSSc and dcSSc.

Totally, $62.5 \%$ of the overlap patients in this study had musculoskeletal manifestations, which confirms previously published data of Balbir-Gurman et al, who reported myositis in $47.5 \%$ of their 40 overlap patients, and Troyanov et al, who reported SSc-myositis overlaps in $42.6 \%$ of their cases. ${ }^{33}$
In this registry, patients with $\mathrm{SSc} /$ myositis, who suffered from muscle weakness together with elevated CK serum levels, 59.1\% had oesophageal involvement, $38.6 \%$ of patients had lung fibrosis, $34.1 \%$ suffered from cardiac involvement, while less than $15.0 \%$ had kidney failure or signs for PH. These frequencies are lower than in the study of Balbir-Gurman who reported higher frequencies of occurrence, but the same trend in the order of frequencies; $84.2 \%$ of patients with SSc-myositis had gastrointestinal involvement, $66.4 \%$ interstitial lung disease, and $26.3 \%$ cardiomyopathy or $\mathrm{PH}$. Most of the patients showing myositis and CK elevation were PmScl positive (22.7\%), followed by 9.1\% anti-U1RNP antibodies, 7.0\% ATA antibodies and $4.5 \%$ ACA, anti-Ku and -Jo1 antibodies. Compared to these data, other groups reported a higher frequency of ATA antibodies. $^{4}$ The frequency of elevated CK serum levels decreased within the course of follow-up visits over a period of 9 years, which could indicate that myositis responds to treatment. ${ }^{34}$ As expected, significantly more SSc-overlap patients have been put on immunosuppressive treatment compared to $1 \mathrm{cSSc}$ and/or dcSSc patients, confirming previously published data. ${ }^{26}$

We found significantly more overlap patients with joint involvement compared to $\mathrm{lcSS}_{\mathrm{c}}$ and dcSSc patients $(\mathrm{p}<0.0001)$, but with no significant difference in the frequency of RF positivity, although the presence of RF and the association with rheumatoid arthritis of SSc patients have been previously discussed controversially. ${ }^{35}$

Regarding the onset of lung fibrosis and heart involvement, patients with SSc-overlap syndromes had an intermediate rate of disease progression in between lcSSc and dcSSc. This observation is further supported by the DLCO levels following a similar course. Overlap patients, in general, had significantly less frequent $\mathrm{PH}$ and kidney involvement than dcSSc patients. The direct comparison between the three major SSc subsets, using Kaplan-Meier curves, visualised that the trend of the SSc-overlap curve was clearly between the curves standing for lcSSc and dcSSc patients (figures 1 and 2). 
Additionally, patients with SSc-overlap syndromes were significantly younger $(48 \pm 1.2$ years) than patients with lcSSc, confirming the data of Garamaschi et al $(48.5 \pm 13.3$ years) and developed less frequently digital ulcers, resulting in significant differences, when compared with dcSSc patients.

Furthermore, the data of this study are in good agreement with Mierau et al and Hasegawa et al, who found that anti-U1RNP antibodies were associated with a younger age of disease onset when using a multiple regression analysis. ${ }^{27} 36$ However, Koschik et al reported that in their patient cohort, patients with PmScl-antibodies were significantly younger than those without PmScl-antibodies. ${ }^{32}$ These patients developed most frequently musculoskeletal involvement, including muscle weakness and synovitis, but in contrast with other studies, less lung fibrosis. ${ }^{37}$ Our study, however, did not allow analysing, whether symptoms and signs of other rheumatic diseases appeared prior or after first SSc features, but Caramaschi et al reported, that $40.5 \%$ of their patients were diagnosed with an additional autoimmune disease prior and $38.1 \%$ after SSc diagnosis. $^{30}$

In summary, we could demonstrate that patients with overlap syndromes differ from lcSSc and dcSSc regarding lung fibrosis and heart involvement, and that musculoskeletal involvement is clearly the most frequent organ manifestation in overlap patients. Although these patients appear to have a milder course of the disease with a mean mRSS similar to lcSSc patients, but less lung fibrosis and heart involvement, when compared to dcSSc patients, they progressed more rapidly with earlier and more widespread significant organ involvement than patients with lcSSc. This study demonstrates that patients with SSc-overlap, on average, carry a higher disease burden than patients with the limited form.

Based on a large cohort, this study strongly supports the idea that patients with SSc-overlap syndromes should be regarded as a separate subset of patients with SSc.

\section{Author affiliations}

'Department of Dermatology, Cologne University Hospital, Cologne, Germany

${ }^{2}$ Department of Dermatology, Medical University of Graz, Graz, Germany

${ }^{3}$ Department of Rheumatology, Asklepios Clinic Altona, Hamburg, Germany

${ }^{4}$ Department of Internal Medicine, Division of Rheumatology, University of Heidelberg, Heidelberg, Germany

${ }^{5}$ Department of Rheumatology, University of Erlangen, Erlangen, Germany ${ }^{6}$ Department of Dermatology, University of Tuebingen, Aachen, Germany

${ }^{7}$ Department of Rheumatology, Clinic of Rheumatology of Aachen, Aachen, Germany

${ }^{8}$ Department of Dermatology, University-Hospital Carl Gustav Carus, Dresden, Germany

${ }^{9}$ Department of Dermatology, Munich University of Technology, Munich, Germany

${ }^{10}$ Department of Rheumatology, University of Tuebingen, Tuebingen, Germany

${ }^{11}$ Institute of Medical Statistics, Informatics and Epidemiology, University of Cologne, Cologne, Germany

${ }^{12}$ Department of Dermatology and Venereology, University of Muenster, Muenster, Germany

${ }^{13}$ Department of Internal Medicine and Nephrology (Centre for interdisciplinary Rheumatology), Robert-Bosch-Hospital, Stuttgart, Germany

${ }^{14}$ Department of Dermatology, Helios Clinic Oberhausen, Oberhausen, Germany

${ }^{15}$ Department of Haemato-Oncology and Rheumatology, University of Heidelberg, Heidelberg, Germany

${ }^{16}$ Department of Rheumatology and Clinical Immunology, Kerckhoff Clinic, Bad Nauheim, Germany

${ }^{17}$ Clinical Research Unit for Rheumatology, University Medical Center Freiburg, Freiburg, Germany

${ }^{18}$ Clinic for Dermatology, Hamburg Alstertal, Hamburg, Germany

${ }^{19}$ Department of Dermatology, Ulm University Hospital, Ulm, Germany

${ }^{20}$ Department of Rheumatology and Clinical Immunology, University of Berlin, Charité, Germany

${ }^{21}$ Department of Dermatology and Allergology, Ludwig Maximilian University, Munich, Germany

${ }^{22}$ Department of Dermatology and Venereology, Ruhr University Bochum, Bochum, Germany
${ }^{23}$ Department of Rheumatology and Clinical Immunology, University Medical Center Freiburg, Freiburg, Germany

${ }^{24}$ Department of Dermatology and Venerology, University of Berlin, Charité, Berlin, Germany

${ }^{25}$ Department of Rheumatology, Johanniter-Hospital, Treuenbrietzen, Germany

Acknowledgements The work of Ebru Keser, Patricia Scholz and Gabriele Browne at the central office in keeping the Network going is gratefully acknowledged.

Collaborators Nil Mona Ahrazoglu, Department of Dermatology, Cologne University Hospital; Mike Oliver Becker, Department of Rheumatology and Clinical Immunology, University of Berlin, Charité; Rudolf Mierau, Department of Rheumatology, Clinic of Rheumatology Aachen; Gunther Neeck, Medical Center for Rheumatology Bad Doberan; Christoph Fiehn, Medical Center for Rheumatology, Baden-Baden; Aaron Juche, Department of Rheumatology, Immanuel Hospital Berlin-Buch; Andrea Rubbert, Department of Rheumatology, University of Cologne; Manfred Weber, Department of Internal Medicine, Hospital Cologne Merheim; Rebecca Fischer-Betz, Department of Nephrology and Rheumatology, University of Duesseldorf; Michael Sticherling, Department of Dermatology and Venereology, University of Erlangen; Harald Burkhardt, Michaela Koehm, Med. Clinic II, Department of Rheumatology, University of Frankfurt; Cornelia S Seitz, Claudia Herink, Department of Dermatology, Venereology and Allergology, University of Goettingen; Ivan Foeldvári, Department of Rheumatology, Schoen Clinic, Hamburg-Eilbek; Johannes Norgauer, Department of Dermatology and Venereology, University of Jena; Dieter Schoeffel, Clinic for Rheumatology, Mannheim; Regina Renner, Mirjana Ziemer, Department of Dermatology and Venereology, University of Leipzig; Kerstin Steinbrink, Department of Dermatology and Venereology, University of Mainz; Rudolf Stadler, Verena Müller, Department of Dermatology and Venereology, Johannes Wesling Hospital, Minden; Mahzad Ziai, Department of Dermatology, Munich University of Technology; Sigrid Karrer, Michael Landthaler Department of Dermatology and Venereology, University of Regensburg; Rotraud Meyringer, Department of Internal Medicine, University of Regensburg; Michael Buslau, Clinic for Rehabilitation Rheinfelden (CH); Dirk Tomsitz, Hae- Hyuk Lee, Department of Dermatology and Venereology, University of Berlin, Charité; Percy Lehmann, Noemi Gaebelein-Wissing, Helios Clinic, Department of Dermatology, Wuppertal; Marieke Wagner, Department of Rheumatology, University of Tuebingen; Joachim Ebel, Department of Rheumatology, Asklepios Clinic Altona; Christian Beyer, Department of Rheumatology, University of Erlangen; Rita Varga, Department of Dermatology and Allergology, Ludwig Maximilian University, Munich; Agnes Bretterklieber, Department of Dermatology, Medical University of Graz; Frank Reichenberger, Department of Pneumology, Asklepios Clinic Gauting,

Muenchen-Gauting; Andrea Himsel, Clinic for Internal Medicine and Rheumatology, GPR MVZ Ruesselsheim

Contributors PM, NH and TK have conceived and designed the study. All authors and coauthors (excluding LH and KK) were involved in data acquisition. LH and KK have made particular contribution to statistical analyses and interpretation of data. All authors contributed to the interpretation of the data and jointly approved the final manuscript.

Funding This study was supported by a grant of the German Federal Ministry of Education and Research (BMBF) (01GM0310) and a grant of the Edith-BuschFoundation. The work of Pia Moinzadeh was supported by a german 'Koeln Fortune' and 'Deutsche Stiftung Sklerodermie' grant.

Competing interests None.

\section{Patient consent Obtained.}

Ethics approval Ethics Committee University of Cologne.

Provenance and peer review Not commissioned; externally peer reviewed.

Open Access This is an Open Access article distributed in accordance with the Creative Commons Attribution Non Commercial (CC BY-NC 4.0) license, which permits others to distribute, remix, adapt, build upon this work non-commercially, and license their derivative works on different terms, provided the original work is properly cited and the use is non-commercial. See: http://creativecommons.org/ licenses/by-nc/4.0/

\section{REFERENCES}

1 Preliminary criteria for the classification of systemic sclerosis (scleroderma). Subcommittee for scleroderma criteria of the American Rheumatism Association Diagnostic and Therapeutic Criteria Committee. Arthritis Rheum 1980;23:581-90.

2 LeRoy EC, Black C, Fleischmajer R, et al. Scleroderma (systemic sclerosis): classification, subsets and pathogenesis. J Rheumatol 1988;15:202-5.

3 Galluccio F, Walker UA, Nihtyanova $S$, et al. Registries in systemic sclerosis: a worldwide experience. Rheumatology (Oxford) 2011;50:60-8.

4 Balbir-Gurman A, Braun-Moscovici Y. Scleroderma overlap syndrome. Isr Med Assoc J 2011:13:14-20.

5 Elhai M, Avouac J, Kahan A, et al. Systemic sclerosis at the crossroad of polyautoimmunity. Autoimmun Rev 2013. 
6 Cappelli S, Bellando Randone S, Martinovic D, et al. "To be or not to be," ten years after: evidence for mixed connective tissue disease as a distinct entity. Semin Arthritis Rheum 2012;41:589-98.

7 Ziswiler HR, Urech R, Balmer J, et al. Clinical diagnosis compared to classification criteria in in a cohort of 54 patients with systemic sclerosis and associated disorders. Swiss Med Wkly 2007;137:586-90.

8 Fiori G, Pignone A, Cerinic MM. Overlap syndromes. Reumatizam 2002;49:12-15.

9 Jury EC, D'Cruz D, Morrow WJ. Autoantibodies and overlap syndromes in autoimmune rheumatic disease. J Clin Pathol 2001;54:340-7.

10 Meier FM, Frommer KW, Dinser $\mathrm{R}$, et al. Update on the profile of the EUSTAR cohort: an analysis of the EULAR Scleroderma Trials and Research group database. Ann Rheum Dis 2012;71:1355-60.

11 Jablonska S, Blaszczyk M. Scleroderma overlap syndromes. Adv Exp Med Biol 1999;455:85-92.

12 Pakozdi A, Nihtyanova S, Moinzadeh P, et al. Clinical and serological hallmarks of systemic sclerosis overlap syndromes. J Rheumatol 2011;38:2406-9.

13 Hashimoto $A$, Endo $\mathrm{H}$, Kondo $\mathrm{H}$, et al. Clinical features of 405 Japanese patients with systemic sclerosis. Mod Rheumatol 2012;22:272-9.

14 laccarino L, Gatto M, Bettio S, et al. Overlap connective tissue disease syndromes. Autoimmun Rev 2013;12:363-73.

15 Jablonska S, Blaszyk M. Scleromyositis (scleroderma/polimyositis overlap) is an entity. J Eur Acad Dermatol Venereol 2004;18:265-6.

16 Szucs G, Szekanecz Z, Zilahi E, et al. Systemic sclerosis-rheumatoid arthritis overlap syndrome: a unique combination of features suggests a distinct genetic, serological and clinical entity. Rheumatology (Oxford) 2007;46:989-93.

17 Ueda-Hayakawa I, Hasegawa M, Kumada S, et al. Usefulness of anti-cyclic citrullinated peptide antibody and rheumatoid factor to detect rheumatoid arthritis in patients with systemic sclerosis. Rheumatology (Oxford) 2010;49:2135-9.

18 Ho KT, Reveille JD. The clinical relevance of autoantibodies in scleroderma. Arthritis Res Ther 2003;5:80-93.

19 Maddison PJ. Overlap syndromes and mixed connective tissue disease. Curr Opin Rheumatol 1991:3:995-1000.

20 Graf SW, Hakendorf P, Lester S, et al. South Australian Scleroderma Register: autoantibodies as predictive biomarkers of phenotype and outcome. Int J Rheum Dis 2012;15:102-9.

21 Steen VD. Autoantibodies in systemic sclerosis. Semin Arthritis Rheum 2005;35:35-42

22 Nihtyanova SI, Denton CP. Autoantibodies as predictive tools in systemic sclerosis. Nat Rev Rheumatol 2010;6:112-16.
23 Cepeda EJ, Reveille JD. Autoantibodies in systemic sclerosis and fibrosing syndromes: clinical indications and relevance. Curr Opin Rheumatol 2004;16:723-32.

24 Oddis CV, Okano Y, Rudert WA, et al. Serum autoantibody to the nucleolar antigen PM-Scl. Clinical and immunogenetic associations. Arthritis Rheum 1992;35:1211-17.

25 Hunzelmann N, Genth E, Krieg T, et al. The registry of the German Network for Systemic Scleroderma: frequency of disease subsets and patterns of organ involvement. Rheumatology (Oxford) 2008;47:1185-92.

26 Hunzelmann N, Moinzadeh P, Genth E, et al. High frequency of corticosteroid and immunosuppressive therapy in patients with systemic sclerosis despite limited evidence for efficacy. Arthritis Res Ther 2009;11:R30.

27 Mierau R, Moinzadeh P, Riemekasten G, et al. Frequency of disease-associated and other nuclear autoantibodies in patients of the German Network for Systemic Scleroderma: correlation with characteristic clinical features. Arthritis Res Ther 2011;13:R172.

28 Alarcon GS. Unclassified or undifferentiated connective tissue disease. Best Pract Res Clin Rheumatol 2000;14:125-37.

29 Poormoghim H, Lucas M, Fertig N, et al. Systemic sclerosis sine scleroderma: demographic, clinical, and serologic features and survival in forty-eight patients. Arthritis Rheum 2000:43:444-51.

30 Caramaschi P, Biasi D, Volpe A, et al. Coexistence of systemic sclerosis with other autoimmune diseases. Rheumatol Int 2007;27:407-10.

31 Hudson $M$, Rojas-Villarraga $A$, Coral-Alvarado $P$, et al. Polyautoimmunity and familial autoimmunity in systemic sclerosis. J Autoimmun 2008;31:156-9.

32 Koschik RW 2nd, Fertig N, Lucas MR, et al. Anti-PM-Scl antibody in patients with systemic sclerosis. Clin Exp Rheumatol 2012;30:S12-16.

33 Troyanov Y, Targoff IN, Tremblay JL, et al. Novel classification of idiopathic inflammatory myopathies based on overlap syndrome features and autoantibodies: analysis of 100 French Canadian patients. Medicine 2005;84:231-49.

34 Burdt MAHR, Deutscher SL, Wang GS, et al. Long-term outcome in mixed connective tissue disease: longitudinal clinical and serologic findings. Arthritis Rheum 1999;42:899-909.

35 Schmeiser T, Pons-Kuhnemann J, Ozden F, et al. Arthritis in patients with systemic sclerosis. Eur J Intern Med 2012;23:e25-9.

36 Hasegawa M, Hatta Y, Matsushita T, et al. Clinical and laboratory features dependent on age at onset in Japanese systemic sclerosis. Mod Rheumatol 2012.

37 Skare TL, Fonseca AE, Luciano AC, et al. Autoantibodies in scleroderma and their association with the clinical profile of the disease. A study of 66 patients from southern Brazil. Anais brasileiros de dermatologia 2011;86:1075-81. 